\title{
PRESSURE PAIN SENSITIVITY: MARKER FOR STRESS AFFECTING GENERAL HEALTH
}

\author{
Václav Vaněček ${ }^{1}$, Milan Tuček ${ }^{1}$, Søren Ballegaard² \\ ${ }^{1}$ Institute of Hygiene and Epidemiology, First Faculty of Medicine, Charles University and General University Hospital, Prague, Czech Republic \\ UII Care, Hellerup, Denmark
}

\section{SUMMARY}

Objective: The study examined the links between pressure pain sensitivity (PPS) measured during preventive medical examination of men, their health status and occupation.

Method: A one year (2015-2016) convenience sampling technique was used to gain primary data from actual medical examination, health records (personal and occupational history) and pressure pain sensitivity (PPS) measured by UII Meter equipment during preventive medical examination of 371 men of different occupations (average age 43.6 \pm 10.4 years, range 19-66 years). Measured PPS values of 60 or more indicate high PPS, contrary PPS values to 40 indicate low PPS.

Results: 345 men (93\%) were considered healthy (without diagnosis of a disease), 26 men (7\%) had positive personal history/symptoms of disease. The average of measured PPS values for the whole group was $36.6 \pm 9.5$ (first measurement) and $36.7 \pm 8.5$ (second, repeated measurement), suggesting a high reproducibility of the measurements $(r=0.80)$. Decreased PPS values were measured in men without reported diagnosis of a disease compared to men with diagnosed diseases. Elevated PPS values were measured in men with symptoms of neurocirculatory asthenia (NCA) compared to asymptomatic men and also to men with different diagnosis. The group of men with other than NCA symptoms did not differ significantly in PPS values compared to group of asymptomatic men. Road drivers (177 men) did not differ significantly compared to other occupations (194 men).

Conclusion: The increased neuropsychological load/stress is connected with increased pain sensitivity to pressure. The PPS method is objective, reliable, simple, and noninvasive evaluation of the impact of stress and may be helpful in assessing medical fitness to work.

Key words: pressure pain sensitivity, stress marker, health impact

Address for correspondence: M. Tuček, Institute of Hygiene and Epidemiology, First Faculty of Medicine, Charles University, Studničkova 7, 12000 Prague 2, Czech Republic. E-mail: milan.tucek@lf1.cuni.cz

https://doi.org/10.21101/cejph.a4931

\section{INTRODUCTION}

Historically, stress has been defined by Selye (1980) as a reaction from a calm state to an excited state for the purpose of preserving the integrity of the organism (1). Stress is defined as an influence which is potentially threatening to the health of the individual. Stress sets off an alarm in the brain, which responds by preparing the body for defensive action: it is essential to distinguish between transient and persistent stress (2). Transient stress is a physiological state of preparedness, a state that is automatically induced through neural/hormonal signals from the brain when a threat or work challenge is perceived. Thus, it serves as a defence mechanism and helps to increase performance level whether it is to be used in a fight/flight situation or to solve a job task. When the threat/challenge is over, homeostasis is reestablished. The process of activation and recovery back to normal levels is also known as allostasis. In contrast, persistent stress occurs due to a prolonged exposure of the hormones involved in transient stress causing an allostatic load, which leads to physiological dysfunctions that can be harmful to health. Since stress is not directly measurable, researchers often use physiological markers (heart rate, blood pressure, hormone levels, etc.) together with behavioural observations and personal questionnaires. The physiological markers are essential since they require a minimum of cognitive and emotional processing and thus are more objective. However, many of these physiological markers are influenced by several other factors than stress, i.e. physical exercise, tobacco and/or alcohol use and the time of a day. During clinical observations of patients with heart diseases and other conditions of stress-related origin, increased pain sensitivity on specific locations on the skin of the sternum has been observed (3). This observation is in concordance with the notion of a hyperalgesic effect and can be explained by the occurrence of cutaneous polymodal nociceptors, which responds to mechanical stimuli, noxious heat, exogenous chemicals, and inflammatory mediators, and might result in a noxious withdrawal reflex (NWR). The NWR is regarded as a reliable and objective tool for exploring pain control systems in humans. In a fight/ flight situation it makes sense to prepare for a maximal defence reaction in order to protect the vital heart region. The existence of points on the sternum with association to the heart is part of oriental acupuncture tradition. On this background, it is possible to hypothesize that transient and persistent stress increases the sensitivity in specific points located in an area of the skin on the 
sternum and that this sensitivity can be measured as pressure pain sensitivity (PPS), which can be a useful marker for stress (3).

\section{MATERIALS AND METHODS}

A one year (2015-2016) convenience sampling technique was used to gain primary data from actual medical examination, health records (personal and occupational history) and pressure pain sensitivity (PPS) measured by Ull Meter equipment during preventive medical examination of 371 men of different occupations (average age $43.6 \pm 10.4$ years, range 19-66 years). PPS was measured with the probands in supine position by an algometric instrument (Ull Meter), which transfers the pain threshold into a logarithmic scale and invert it into a sensitivity scale (high value $=$ low threshold). Each trial started with the introduction of technique with two measurements on the dorsal part of the middle phalanx on the left index finger. The measuring device was applied with a gradually increase of pressure, allowing 3-4 seconds pressure time. The person was instructed to say "Stop" as soon as discomfort was felt. If the researcher observed a startle or withdrawal reflex this was also considered as a stop signal. Subsequently, measurements on the sternum were conducted following the same procedure. The point for measuring was identified by palpation as the most tender point of the skin on the sternum within the area between the third, fourth and fifth left intercostal space, reflecting the area of segmental innervation of the heart (5). The PPS method was previously described in detail (4) (Fig. 1). Differences between group PPS values were tested by t-test (p values) (Table 1 ).

\section{RESULTS}

The authors present the results of measurements of PPS in a group of 371 men of average age $43.6 \pm 10.4$ years (range 19-66

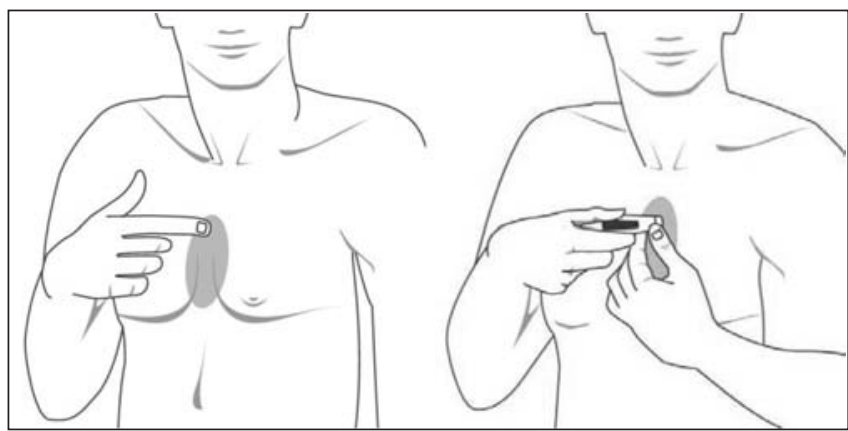

Fig. 1. PPS measuring.

1. Search the area between the chest bone and the rib with your finger.

2. Find the most sensitive point.

3. Turn on the instrument.

4. Place the instrument pad perpendicular to the sensitive point, and support it with one hand.

5. With the other hand, the pressure is now increased over 2-3 seconds until starting soreness/discomfort of the person being measured on, or until the instrument alarm tone, then you must remove the instrument straight away. 6. Now read the measurement result on the instrument display.

years), of which 345 men (93\%) could be considered healthy (without diagnosis of a disease) and 26 men (7\%) had positive personal history/symptoms of disease (main diagnoses: neurocirculatory asthenia (NCA) ten times; diabetes mellitus four times; vertebrogenic algic syndrome and cardiovascular disease three times; pain syndrome after injury two times; and chronic progressive polyarthritis, Crohn's disease, peptic ulcer disease and bronchial asthma once). Average of measured PPS values for the whole group was $36.6 \pm 9.5$ (first measurement) and $36.7 \pm 8.5$ (second, repeated measurement), suggesting a high reproducibility of the measurements $(r=0.80)$ (Fig. 2).

Summary results are presented in Table 1 . The PPS values in men without reported disease were statistically significantly different in comparison with men with diagnosed diseases (PPS values $35.8 \pm 8.4,35.5 \pm 5.9$ vs. $45.1 \pm 15.4,52.4 \pm 19.8$, respectively, p value of the first measurement 0.001 , of the second measure-

Table 1. Summary table of group PPS values ( $N=371 \mathrm{men})$

\begin{tabular}{|c|c|c|c|c|c|c|c|c|c|}
\hline & \multirow{2}{*}{ Group } & \multirow{2}{*}{$n$} & \multicolumn{2}{|c|}{ PPS value } & \multirow{2}{*}{ Group } & \multirow{2}{*}{$\mathrm{n}$} & \multicolumn{2}{|c|}{ PPS value } & \multirow{2}{*}{$\begin{array}{c}\text { Probability } \\
\text { p value } \\
\text { (t-test) }\end{array}$} \\
\hline & & & Aritm. mean & SD & & & Aritm. mean & SD & \\
\hline 1st measure & Drivers & 177 & 35.7 & 9.4 & Other occupations & 194 & 36.5 & 9.5 & 0.998 \\
\hline 2nd measure & Drivers & 177 & 36.4 & 10.9 & Other occupations & 194 & 37.8 & 11.4 & 0.640 \\
\hline 1st measure & "Healthy" & 345 & 35.8 & 8.4 & With disease & 26 & 45.1 & 15.4 & 0.001 \\
\hline 2nd measure & "Healthy" & 345 & 35.5 & 5.9 & With disease & 26 & 52.4 & 19.8 & $<0.001$ \\
\hline 1st measure & NCA & 10 & 50.8 & 14.8 & Other dg & 16 & 38.8 & 13.3 & 0.015 \\
\hline 2nd measure & NCA & 10 & 67.3 & 11.4 & Other $\mathrm{dg}$ & 16 & 43.4 & 19.7 & 0.001 \\
\hline 1st measure & NCA & 10 & 50.8 & 14.8 & "Healthy" & 345 & 35.8 & 8.4 & 0.002 \\
\hline 2nd measure & NCA & 10 & 67.3 & 11.4 & "Healthy" & 345 & 35.5 & 5.9 & $<0.001$ \\
\hline 1st measure & Other dg & 16 & 38.8 & 13.3 & "Healthy" & 345 & 35.8 & 8.4 & 0.131 \\
\hline 2nd measure & Other dg & 16 & 43.4 & 19.7 & "Healthy" & 345 & 35.5 & 5.9 & 0.116 \\
\hline 1st measure & $\Sigma$ & 371 & 36.6 & 9.5 & & & & & \\
\hline 2nd measure & $\Sigma$ & 371 & 36.7 & 8.5 & & & & & \\
\hline
\end{tabular}

PPS - pressure pain sensitivity, SD - standard deviation, $n$ - number of examined persons, $\mathrm{dg}$ - diagnosis, NCA - neurocirculatory asthenia 


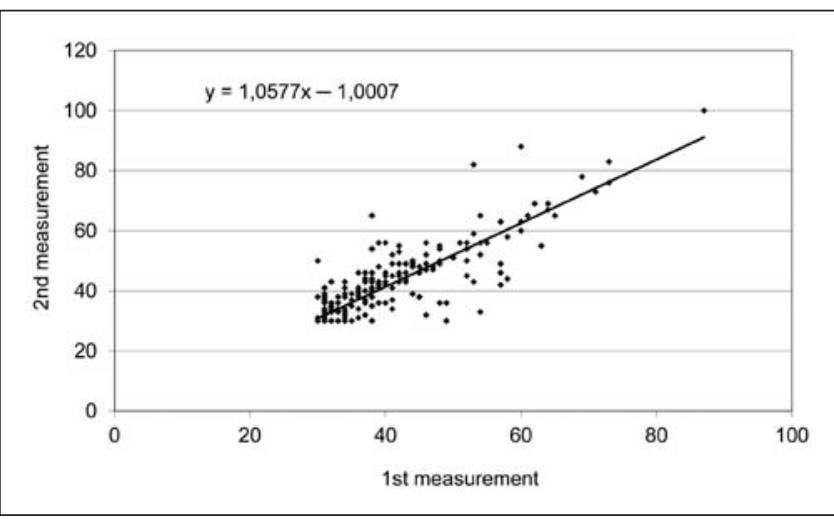

Fig. 2. PPS measurement values reproducibility (371 men).

ment less than 0.001). The measured PPS values in men with NCA symptoms were statistically significantly different compared to asymptomatic men (PPS values $50.8 \pm 14.8$, 67.3 \pm 11.4 vs. $38.8 \pm 13.3$, $35.5 \pm 5.9$, respectively, p value of the first measurement 0.002 , of the second measurement less than 0.001) and also compared to a group of men with different diagnosis (PPS values $50.8 \pm 14.8,67.3 \pm 11.4$ vs. $38.8 \pm 13.3,43.4 \pm 19.7$, respectively, p value of the first measurement 0.015 , of the second measurement 0.001). The group of men with other than NCA symptoms did not differ significantly in PPS values compared to a group of asymptomatic men. Road drivers (177 men, PPS average value $35.7 \pm 9.4,36.4 \pm 10.9$, respectively) did not differ significantly compared to other occupations (194 men, PPS average value $36.5 \pm 9.5$, $37.8 \pm 11.4$, respectively). There was no association between measured PPS values and age.

\section{DISCUSSION}

Convenience data sampling as a specific type of method relies on data collection from population members who are conveniently available to participate in a study. This technique may prove to be effective during exploration stage of the research area. Authors are aware of disadvantages of convenience sampling technique (vulnerability to selection bias and influences beyond the control of the researcher, possibility of sampling error and restricted credibility). The employees/workers undergo preventive medical occupational examinations for the evaluation of health status to be protected against occupational and work-related diseases. Those examined persons are in most cases formally "healthy", however, they might experience stress due to the necessity to undergo medical examination and some of them have different symptoms of diseases (6).

Measured PPS values of 60 or more indicate high PPS, contrary, PPS values to 40 indicate low PPS. Because in "healthy" persons low PPS values were measured and elevated PPS values were measured only in "unhealthy" persons, it can be concluded that the necessity to undergo medical examination was not stressful. Persistent stress may affect negatively work performance, general health and so medical fitness for work. It seems to be associated with the development of elements of the metabolic syndrome, as well as ischaemic heart disease, type 2 diabetes, depression, and post-traumatic stress syndrome (PTSD). Neurocirculatory asthenia is usually defined as a psychosomatic disorder characterized by nervous and circulatory irregularities (dyspnea, palpitation, giddiness, vertigo, tremor, precordial pain, and increased susceptibility to fatigue) resulting from or associated with psychological stress. Recent studies suggest a causal association between PPS and a variety of important cardiovascular physiological factors and metabolic syndrome characteristics associated with persistent stress. PPS may be able to identify a group of office workers who will benefit from a stress-reducing intervention programme (3). The study on women with breast cancer examined before and after 6 months of intervention provided clear evidence for the recommendation of the PPS measure as a marker for persistent stress (2).

\section{CONCLUSSIONS}

Based on the PPS measurement results, it may be preliminary concluded that the increased neuropsychological load/stress is also connected with increased pain sensitivity to pressure, which is quite clearly reflected in the examined men with neuropsychological symptoms mostly reported in personal history (neurocirculatory asthenia and/or "stressful" disease in general). The applicability of this method for objective, reliable, simple, and noninvasive evaluation of the impact of stress is necessary to continue to refine, with emphasis on its potential for health promotion intervention programme. The method of measurement of PPS may also help to refine the diagnosis of the neuropsychological disorders, which could be helpful in assessing medical fitness to work in certain safety related occupations (car or train drivers).

\section{Acknowledgements}

The study was supported by the programme PRVOUK P25/LF1/2, Charles University, Prague.

\section{Conflict of Interests}

None declared

\section{REFERENCES}

1. Selye H, editor. Selye's guide to stress research. Volume 1. New York: Van Nostrand Reinhold; 1980.

2. Axelsson CK, Ballegaard S, Karpatschof B, Schousen P. Pressure pain sensitivity as a marker for stress and pressure pain sensitivity-guided stress management in women with primary breast cancer. Scand J Clin Lab Invest. 2014 Aug;74(5):399-407.

3. Ballegaard S, Petersen PB, Harboe GS, Karpatschof B, Gyntelberg F, Faber J. The association between changes in pressure pain sensitivity and changes in cardiovascular physiological factors associated with persistent stress. Scand J Clin Lab Invest. 2014 Mar;74(2):116-25.

4. Ballegaard S, Karpatschof B, Trojaborg W, Hansen AM, Magnusson G, Petersen PB. A simple and objective marker for stress. Scand J Clin Lab Invest. 2009;69(6):713-21.

5. Magnusson G, Ballegaard S, Karpatschof B, Nyboe J. Long-term effects of integrated rehabilitation in patients with stroke: a nonrandomized comparative feasibility study. J Altern Complement Med. 2010 Apr;16(4):36974.

6. Tuček M. Transport safety, neurobehavioral disorders and medical fitness standards. Neural Network World. 2010;20(6):723-36. 